\title{
LEBESGUE MEASURE IS A REPRESENTING MEASURE ${ }^{1}$
}

\section{S. J. SIDNEY}

ABSTRACT. Lebesgue measure on the unit interval $I$ is multiplicative on some maximal Dirichlet algebra on $I$. Related results are obtained.

The main point of the present note is the observation that Lebesgue measure on the unit interval $I=[0,1]$ is multiplicative on some uniform algebra on $I$, which answers a question which has apparently circulated for some time, and was posed to me by my colleague G. M. Leibowitz.

Theorem. If $\mu$ is a nonatomic (Borel) probability measure on I whose closed support is all of $I$, then $\mu$ is multiplicative on some maximal (proper) Dirichlet subalgebra of $C(I)$.

Proof. If $J$ is an arc in the complex plane $\mathrm{C}$, we consider the algebra $A$, first studied by $\mathrm{J}$. Wermer [4], of functions continuous on the Riemann sphere $S^{2}=\mathrm{C} \cup\{\infty\}$ and holomorphic on $U=S^{2} \backslash J$. It has been shown by A. Browder and J. Wermer [1] that $J$ can be so chosen that $A$ is a uniform algebra whose Šilov boundary is $J$, and $A \mid J$ is a maximal (proper) Dirichlet algebra on $J$. Pick $z$ in $U$ and let $\nu$ denote the representing measure for $z$ on $J$. Then $\nu$ is nonatomic, since any atom of $\nu$ would lie in the Gleason part for $A$ which contains $z$, whereas all points of $J$ are peak points for $A$. Further, the closed support of $\nu$ is all of $J$. For let $x \in J$ and let $V$ be an open set in $S^{2}$ containing $x$. There is $f$ in $A$ such that $f(x)=\sup |f|=1$ while $|f|<1 / 3$ on $S^{2} \backslash V$. Take $z^{\prime} \in U$ so close to $x$ that $\left|f\left(z^{\prime}\right)\right|>2 / 3$. If $\nu^{\prime}$ denotes the representing measure for $z^{\prime}$ on $J$, then $\nu$ and $\nu^{\prime}$ are (boundedly) equivalent measures, because $z$ and $z^{\prime}$ lie in the same Gleason part for $A$. But clearly $\int f d \nu^{\prime}=f\left(z^{\prime}\right)$ implies that $\nu^{\prime}$, hence $\nu$, has some mass on $J \cap V$.

Received by the editors May 17, 1973.

AMS (MOS) subject classifications (1970). P'rimary 46J10; Secondary 46J15, $30 \mathrm{~A} 98$.

Key words and phrases. Representing measure, uniform algebra, multiplicative, Dirichlet algebra, Gleason part, peak point, Jensen measure.

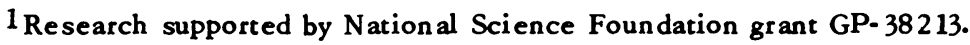


Let $\tau$ denote a homeomorphism of $I$ onto $J$. Define functions $g, h$ : $I \rightarrow I$ by $g(t)=\nu(f([0, t]))$ and $h(t)=\mu([0, t])$. These are homeomorphisms of $I$ onto itself, and $\mu$ is multiplicative on the maximal Dirichlet algebra $\left\{f \circ \tau \circ g^{-1} \circ h: f \in A\right\}$ on $I$. Q.E.D.

Thus Lebesgue measure is even a Jensen measure.

The heart of the above argument is existence of a nonatomic multiplicative measure $\nu$ whose support is precisely $J$. As the following theorem shows, this existence can be recovered if we know simply that $J$ is the Šilov boundary for $A$, which happens, e.g., if $J$ has locally positive measure (cf. [3, 7.9]); of course, this entails replacing "maximal Dirichlet" by "uniform" in the statement of the preceding theorem, though the measure will remain an Arens-Singer measure because $A$ is known to satisfy $A^{-1}=$ $\exp (A)$.

Theorem. Let $A$ be a uniform algebra on the compact metric space $X$ and let $\pi$ be a Gleason part. for $A$ which is not contained in X. Then any $z \in \pi$ has a nonatomic representing measure on $X$ whose closed support contains every peak point for $A$ which lies in the closure of $\pi \backslash X$.

Proof. Let $\left\{z_{n}\right\}$ denote a dense sequence in $\pi \backslash X$ (repetitions allowed in case $\pi \backslash X$ is finite). There are (strictly) positive constants $b_{n}$ such that $u(z)-b_{n} u\left(z_{n}\right) \geq 0$ whenever $u \in \operatorname{Re}(A)$ is nonnegative, so by Choquet's theorem (cf. [2]) there is a positive (Borel) measure $\sigma_{n}$ on $X$ supported by $P$, the set of peak points for $A$, such that

$$
\int f d \sigma_{n}=f(z)-b_{n} f\left(z_{n}\right)
$$

for every $f \in A$. Let $\nu_{n}$ be a Jensen measure for $z_{n}$ on $X$, i.e., a representing measure such that $\log \left|f\left(z_{n}\right)\right| \leq \int \log |f| d \nu_{n}$ for all $f \in A$. It is immediate that $\nu_{n}$ is nonatomic.

The measure $\nu=\Sigma_{1}^{\infty} 2^{-n}\left(\sigma_{n}+b_{n} \nu_{n}\right)$ is, by (1), a representing measure for $z$. If it had an atom $x$, then $x$ and $z$ would lie in the same Gleason part for $A$; on the other hand, since the $\nu_{n}$ are nonatomic, $x$ would be an atom for some $\sigma_{n}$, hence $x \in P$, a contradiction. Thus $\nu$ is nonatomic. Finally, let $x \in P$ lie in the closure of $\pi \backslash X$. If $V$ is a neighborhood of $x$ in the spectrum of $A$, we can argue as in the proof of the preceding theorem to see that for $z_{n}$ close to $x, \nu_{n}$ (and so $\nu$ ) has some mass on $X \cap V$. Thus $\nu$ has all the required properties. Q.E.D.

If $X$ is not metrizable, the theorem will still hold provided $\pi \backslash X$ is separable, or at least contains a sequence whose closure contains all 
(generalized) peak points lying in the closure of $\pi \backslash X$. In this case $\sigma_{n}$ is selected to be a "maximal" measure (cf. [2]) and some extra care is required in working out the details.

\section{REFERENCES}

1. A. Browder and J. Wermer, Some algebras of functions on an arc, J. Math. Mech. 12 (1963), 119-130. MR $26 \# 1770$.

2. R. R. Phelps, Lectures on Choquet's theorem, Van Nostrand, Princeton, N. J., 1966. MR 33 \# 1690.

3. E. L. Stout, The theory of uniform algebras, Bogden \& Quigley, Tarrytownon-Hudson, N. Y., 1971.

4. J. Wermer, Polynomial approximation on an arc in $C^{3}$, Ann. of Math. (2) 62 (1955), 269-270. MR 17, 255.

DEPARTMENT OF MATHEMATICS, UNIVERSITY OF CONNECTICUT, STORRS, CONNECTICUT 06268 